\title{
Effects of coenzyme Q10 supplementation on antioxidant capacity and inflammation in hepatocellular carcinoma patients after surgery: a randomized, placebo-controlled trial
}

Hsiao-Tien Liu ${ }^{1,2}$, Yi-Chia Huang ${ }^{3}$, Shao-Bin Cheng ${ }^{1,2}$, Yin-Tzu Huang ${ }^{2}$ and Ping-Ting Lin 3,4* $^{3}$

\begin{abstract}
Background: It has been reported that higher levels of oxidative stress and inflammation play a key role in the progression of hepatocellular carcinoma (HCC) after surgery. Coenzyme Q10 is an endogenous lipid-soluble antioxidant. To date, no intervention study has investigated coenzyme Q10 supplementation in HCC patients after surgery. The purpose of this study was to investigate oxidative stress, antioxidant enzymes activity, and inflammation levels in HCC patients after surgery following administration of coenzyme Q10 (300 mg/day).
\end{abstract}

Methods: This study was designed as a single-blinded, randomized, parallel, placebo-controlled study. Patients who were diagnosed with primary HCC $(n=41)$ and were randomly assign to a placebo $(n=20)$ or coenzyme Q10 (300 mg/day, $n=21)$ group after surgery. The intervention lasted for 12 weeks. Plasma coenzyme Q10, vitamin $\mathrm{E}$, oxidative stress antioxidant enzymes activity and inflammatory markers levels were measured.

Results: The oxidative stress $(p=0.04)$ and inflammatory markers (hs-CRP and IL-6, $p<0.01$ ) levels were significantly decreased, and the antioxidant enzymes activity was significantly increased $(p<0.01)$ after 12 weeks of coenzyme Q10 supplementation. In addition, the coenzyme Q10 level was significantly negatively correlated with the oxidative stress $(p=0.01)$, and positively correlated with antioxidant enzymes activity (SOD, $p=0.01 ; \mathrm{CAT}, p<0.05 ; \mathrm{GPx}, p=0.04)$ and vitamin E level ( $p=0.01)$ after supplementation.

Conclusion: In conclusion, we demonstrated that a dose of $300 \mathrm{mg} / \mathrm{d}$ of coenzyme Q10 supplementation significantly increased the antioxidant capacity and reduced the oxidative stress and inflammation levels in HCC patients after surgery.

Trial registration: Clinical Trials.gov Identifier: NCT01964001

Keywords: Coenzyme Q10 supplementation, Oxidative stress, Antioxidation, Inflammation, Hepatocellular carcinoma, Surgery

\footnotetext{
* Correspondence: apt810@csmu.edu.tw

${ }^{3}$ Department of Nutrition, Chung Shan Medical University, Taichung 40201,

Taiwan

${ }^{4}$ Department of Nutrition, Chung Shan Medical University Hospital, Taichung

40201, Taiwan

Full list of author information is available at the end of the article
} 


\section{Background}

The most recent World Health Organization (WHO, 2014) report indicated that liver cancer is the second leading cause of cancer deaths worldwide, and 0.8 million people (9.1\% of the global population) died from liver cancer [1]. In Taiwan, liver cancer is also the second leading cause of cancer deaths among men and women (Ministry of Health and Welfare, 2014). Hepatocellular carcinoma (HCC) accounts for most liver cancers [2]. The major risk factors for HCC include hepatitis $\mathrm{B}$ or $\mathrm{C}$ virus, alcoholic liver disease, and most likely, nonalcoholic fatty liver disease [2]. Recently, it has been reported that higher level of oxidative stress and inflammation play a key role in the progression of HCC [3]. Oxidative stress may increase via the generation of reactive oxygen species (ROS) and defects in redox defense mechanisms with antioxidant enzymes, such as superoxide dismutase (SOD), catalase (CAT), or glutathione peroxidase (GPx), in mitochondria [4]. This mitochondrial dysfunction may lead to pathological mechanisms of chronic hepatic inflammation and subsequent hepatocarcinogenesis [3]. A case-control study has recently found that patients with $\mathrm{HCC}$ had significantly higher levels of oxidative stress, inflammation, and lower antioxidant capacities [5]. Consequently, it is worth trying an agent that can lower oxidative stress and reduce inflammation in patients with HCC.

Coenzyme Q10 (also called ubiquinone) is a lipidsoluble benzoquinone that has 10 isoprenyl units in its side chain and is a key component of the mitochondrial respiratory chain for adenosine triphosphate synthesis $[6,7]$. Additionally, coenzyme Q10 is an intracellular antioxidant that protects membrane phospholipids, mitochondrial membrane protein, and LDL-C from free radical-induced oxidative damage $[8,9]$. In vivo studies have demonstrated that coenzyme Q10 has therapeutic and chemotherapeutic effects by modulating the expression of hepPar-1, alpha-fetoprotein, inducible nitric oxide synthase, cyclooxygenase- 2 and nuclear factor- $\mathrm{kB}$ $(\mathrm{NF}-\kappa \mathrm{B})$ in the liver tissue of rats with $\mathrm{HCC}[10,11]$. However, to date, no published study has investigated the anti-oxidative and anti-inflammation effects of coenzyme Q10 supplementation in patients with HCC. Thus, the purpose of this clinical study was to examine the levels of oxidative stress, antioxidant enzymes activity, and inflammation after coenzyme Q10 supplementation (300 mg/day) in patients with HCC.

\section{Methods}

\section{Participants}

This study was designed as a single-blinded, randomized, parallel, placebo-controlled study. Following tumor resection, patients who were diagnosed with primary HCC (International Classification of Diseases 9, code 155.0) were recruited from the Division of General Surgery of Taichung Veterans General Hospital. We excluded patients who were younger than 20 years of age or older than 80 years of age, as well as during pregnant or lactating women, patient undergoing chemotherapy or hormone therapy, those with a history or current diagnosis of cardiovascular or renal disease, and those taking warfarin or statin medications. For patients taking vitamins supplements, we asked them to stop taking the supplements for at least for 1 month before enrolling in the study. Informed consent was obtained from each subject. This study was approved by the Institutional Review Board of Taichung Veterans General Hospital, Taiwan and registered at Clinical Trials.gov (NCT01964001).

Using a sample size calculation, we expected that the change in the levels of malondialdehyde would be 0.15 $\pm 0.2 \mu \mathrm{M}$ after the coenzyme Q10 supplementation; therefore, the desired power was set at 0.8 to detect a true effect and at an $\alpha$ value equal to 0.05 , with a minimum of 16 subjects in each intervention group. We enrolled $41 \mathrm{HCC}$ patients in this study, and we used a random numbers table to randomly assign the subjects to the placebo $(n=20)$ or coenzyme Q10 (Q10-300 group, $n=21$ ) group. The coenzyme Q10 and placebo (starch) capsules were commercially available preparations (New Health Co., Ltd. Taichung, Taiwan). The intervention was administered for 12 weeks. The subjects were instructed to take two capsules daily (coenzyme Q10 supplements $300 \mathrm{mg} / \mathrm{d}, 150 \mathrm{mg} / \mathrm{b} . \mathrm{i} . \mathrm{d})$. To monitor compliance, we determined the degree of compliance for each patient, according to the number of returning capsules, and we measured the coenzyme Q10 level every 4 weeks after the supplementation. The following data were recorded for all subjects: age, blood pressures, and smoking and drinking habits, and exercise frequency. Body weight, height, waist and hip circumferences were measured; and the body mass index (BMI) and the ratio of waist to hip were calculated.

\section{Blood collection and biochemical measurements}

Fasting venous blood samples $(15 \mathrm{~mL})$ were obtained to estimate each patient's hematological and vitamin status. Blood specimens were collected in Vacutainer tubes (Becton Dickinson, Rutherford, NJ, USA) that contained EDTA as an anticoagulant or that contained no anticoagulant as required. Serum and plasma were prepared after centrifugation (3000 rpm, $4{ }^{\circ} \mathrm{C}, 15 \mathrm{~min}$ ) and were then stored at $-80{ }^{\circ} \mathrm{C}$ until analysis. Hematological entities, such as blood urea nitrogen, creatinine, glutamic oxaloacetic transaminase, glutamic pyruvate transaminase (GPT), and lipid profiles were measured using an automated biochemical analyzer (Hitachi-7180E, Tokyo, Japan). The level of high sensitivity C-reactive protein 
(hs-CRP) was quantified by particle-enhanced immunonephelometry with an image analyzer (Dade Behring, IL, USA). Plasma tumor necrosis factor- $\alpha$ (TNF- $\alpha$ ) (R\&D Systems Inc., Minneapolis, USA) and interleukin6 (IL-6) (eBioscience, CA, USA) levels were measured using an enzyme-linked immunosorbent assay (ELISA) with commercially available kits, according to the manufacturer's instructions.

Plasma coenzyme Q10 and vitamin E levels were measured using high-performance liquid chromatography (HPLC) and were detected with a UV detector at $275 \mathrm{~nm}$ and $292 \mathrm{~nm}$, respectively [12, 13]. Plasma malondialdehyde (MDA) was determined using the TBARs (thiobarbituric acid reactive substances) method, as described by Botsoglou [14]. The red blood cell (RBC) samples were washed with normal saline after removing the plasma. Then, the RBC samples were diluted with $25 \mathrm{x}$ sodium phosphate buffer for superoxide dismutase (SOD) and glutathione peroxidase (GPx) measurements, with $250 x$ sodium phosphate buffer for the catalase (CAT) measurement. The antioxidant enzymes activities (CAT, SOD, and GPx) were determined in the fresh samples and the methods used to measure these activities have been previously described [15-17]. The protein content of the plasma and RBC was determined based on the biuret reaction of the bicinchoninic acid (BCA) kit (Thermo, Rockford, IL, USA). The values of the antioxidant enzymes activities were expressed as unit/ $\mathrm{mg}$ of protein. All analyses were performed in duplicate.

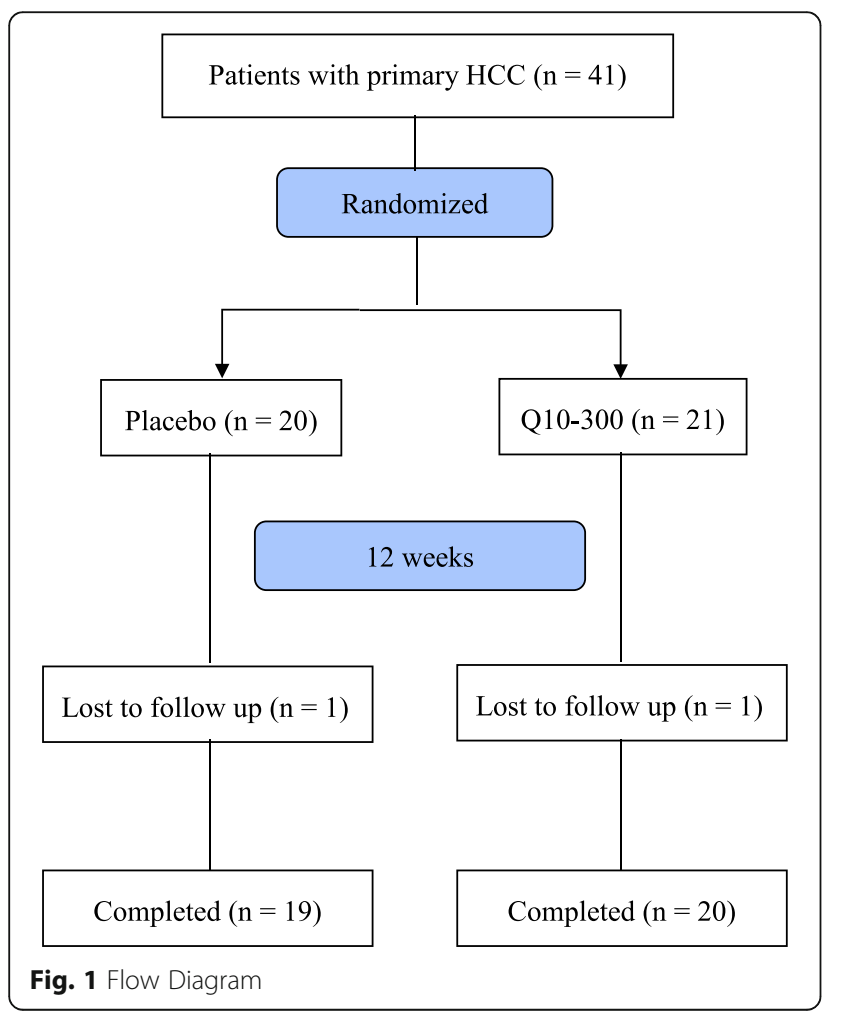

Table 1 Characteristics of subjects

\begin{tabular}{|c|c|c|}
\hline & Placebo $(n=19)$ & Q10-300 $(n=20)$ \\
\hline males $(n, \%)$ & $12(63.2 \%)$ & $15(75.0 \%)$ \\
\hline age (y) & $61.5 \pm 11.0(62.0)^{\mathrm{a}}$ & $59.7 \pm 8.3(58.5)$ \\
\hline $\mathrm{SBP}(\mathrm{mmHg})$ & $133.8 \pm 18.0(134.0)$ & $124.8 \pm 19.3(119.5)$ \\
\hline $\mathrm{DBP}(\mathrm{mmHg})$ & $77.9 \pm 11.2(78.0)$ & $76.9 \pm 12.3(76.0)$ \\
\hline $\begin{array}{l}\text { waist circumference } \\
\text { (cm) }\end{array}$ & $89.1 \pm 7.8(88.0)$ & $86.7 \pm 10.2(88.5)$ \\
\hline waist hip ratio & $0.92 \pm 0.05(0.91)$ & $0.94 \pm 0.16(0.94)$ \\
\hline BMI $\left(\mathrm{kg} / \mathrm{m}^{2}\right)$ & $24.7 \pm 3.2(24.5)$ & $23.1 \pm 2.6(22.8)$ \\
\hline current smoker ${ }^{\mathrm{b}}, n(\%)$ & $6(31.6 \%)$ & $5(25.0 \%)$ \\
\hline drinks alcohol ${ }^{c}, n(\%)$ & $3(15.8 \%)$ & $3(15.0 \%)$ \\
\hline Exercise $^{d}, n(\%)$ & $9(47.4 \%)$ & $8(40.0 \%)$ \\
\hline Hepatitis B, n (\%) & $14(73.7 \%)$ & $12(60.0 \%)$ \\
\hline Hepatitis C, n (\%) & $4(21.1 \%)$ & 7 (35.0 \%) \\
\hline Cirrhosis, $n(\%)$ & $6(31.6 \%)$ & $5(25.0 \%)$ \\
\hline Recurrence, $n(\%)$ & $3(15.8 \%)$ & $4(20.0 \%)$ \\
\hline \multicolumn{3}{|l|}{ Hematology } \\
\hline BUN (mg/dL) & $15.5 \pm 4.9(15.0)$ & $17.1 \pm 9.2(15.5)$ \\
\hline creatinine (mg/dL) & $0.8 \pm 0.3(0.8)$ & $0.9 \pm 0.3(0.9)$ \\
\hline GOT (IU/L) & $35.2 \pm 24.8(27.0)$ & $40.4 \pm 23.0(31.5)$ \\
\hline GPT (IU/L) & $44.8 \pm 36.6(38.0)$ & $55.5 \pm 39.8(45.5)$ \\
\hline $\mathrm{TC}(\mathrm{mg} / \mathrm{dL})$ & $162.7 \pm 36.4(148.0)$ & $162.1 \pm 32.7(156.0)$ \\
\hline TG (mg/dL) & $95.3 \pm 42.3(87.0)$ & $93.4 \pm 29.9(92.5)$ \\
\hline LDL-C (mg/dL) & $100.4 \pm 32.3(90.0)$ & $102.3 \pm 32.8(100.0)$ \\
\hline $\mathrm{HDL}-\mathrm{C}(\mathrm{mg} / \mathrm{dL})$ & $50.6 \pm 11.6(50.0)$ & $49.2 \pm 12.4(47.5)$ \\
\hline $\mathrm{TC} / \mathrm{HDL}-\mathrm{C}$ & $3.3 \pm 1.0(2.9)$ & $3.5 \pm 1.0(3.5)$ \\
\hline \multicolumn{3}{|l|}{ Dietary intake } \\
\hline Energy (kcal/d) & $1608.5 \pm 334.5(1531.2)$ & $1686.5 \pm 460.8(1655.8)$ \\
\hline Protein $(\mathrm{g} / \mathrm{d})$ & $65.9 \pm 17.9(68.0)$ & $66.2 \pm 21.2(66.8)$ \\
\hline$\%$ of total calories & $16.6 \%$ & $15.7 \%$ \\
\hline Fat (g/d) & $49.5 \pm 23.9(48.2)$ & $50.6 \pm 16.1(51.7)$ \\
\hline$\%$ of total calories & $26.6 \%$ & $26.8 \%$ \\
\hline carbohydrate (g/d) & $228.6 \pm 40.8(227.0)$ & $251.1 \pm 89.6(238.6)$ \\
\hline$\%$ of total calories & $57.2 \%$ & $57.9 \%$ \\
\hline PUFA (g/d) & $15.6 \pm 11.8(13.0)$ & $13.8 \pm 8.6(11.1)$ \\
\hline MUFA (g/d) & $15.1 \pm 9.5(11.9)$ & $15.3 \pm 6.6(15.6)$ \\
\hline $\mathrm{SFA}(\mathrm{g} / \mathrm{d})$ & $12.8 \pm 8.0(10.3)$ & $14.2 \pm 5.7(14.3)$ \\
\hline vitamin A ( $\mu \mathrm{g}$ R.E./d) & $\begin{array}{l}3216.8 \pm 4244.7 \\
(1188.1)\end{array}$ & $\begin{array}{l}3204.2 \pm 3705.5 \\
(1110.4)\end{array}$ \\
\hline vitamin $E(m g$ a-T.E./d) & $421.6 \pm 727.1(21.5)$ & $615.0 \pm 989.3(10.8)$ \\
\hline cholesterol (mg/d) & $250.0 \pm 163.8(238.2)$ & $231.8 \pm 117.6$ (197.8) \\
\hline dietary fiber $(g / d)$ & $13.0 \pm 6.5(11.9)$ & $10.9 \pm 6.1(9.7)$ \\
\hline
\end{tabular}

$B M I$ body mass index, BUN blood urea nitrogen, GOT glutamic oxaloacetic transaminase, HCC hepatocellular carcinoma, HDL-C high-density lipoprotein-cholesterol, LDL-C low density lipoprotein-cholesterol, MUFA monounsaturated fatty acid, PUFA polyunsaturated fatty fat, SFA saturated fatty acid, TC total cholesterol, TG triglyceride ${ }^{\text {a }}$ means \pm SD (medians)

${ }^{b}$ current smoker: individuals currently smoking one or more cigarettes per day 'drinks alcohol: individuals drinking one or 1 more alcoholic drinks per day regularly dexercise: individuals who exercise regularly at least 3 times every week 


\section{Statistical analyses}

The data were analyzed using SigmaPlot software (version 12.0, Systat, San Jose, CA, USA). The normality of the distribution of the variables was evaluated using the Shapiro-Wilk test. When comparing the continuous variables between the placebo and the Q10-300 groups, Student's $t$-test or the Mann-Whitney rank sum test was used. For categorical response variables, the differences between the two groups were assessed using the Chi-square test or Fisher's exact test. When comparing the levels of coenzyme Q10, vitamin E, and antioxidant enzymes activities at week $0,4,8$, and week 12 within the group, one-way repeated measures ANOVA or Friedman repeated measures ANOVA on ranks was performed, and used Holm-Sidak post hoc test to assess the statistically significant differences. When comparing the levels of inflammatory markers at week 0 and week 12 within the group, paired- $t$ test was performed. To examine the correlation between the coenzyme Q10 level and the levels of vitamin E, antioxidant enzymes activities, and inflammatory markers after the supplementation, Spearman rank order correlation was used. Additionally, simple linear regressions were used to examine the correlations between the changes in the levels of coenzyme Q10 and the changes in the levels of oxidative stress, antioxidant enzymes activity, and vitamin E. The results were considered to be statistically significant at $P<0.05$. The values presented in the text are the means \pm standard deviation (medians).

\section{Results}

Characteristics and dietary intake of subjects

The sampling and trial profiles are summarized in Fig. 1, along with the number of subjects who completed the study in each group. Thirty-nine subjects completed the intervention (placebo, $n=19$; Q10-300, $n=20$ ). Table 1 shows the demographic characteristics and the dietary intakes of the subjects. At baseline, there were no significant between group differences in terms of age, blood pressure, anthropometric measurements, hematological entities, and dietary intake, as well as the frequency of smoking, drinking, or exercise. A total of $66.7 \%$ of the subjects had hepatitis $B$, and $28.2 \%$ of the subjects had hepatitis B or cirrhosis. In addition, $82.0 \%$ of the subjects were newly diagnosed with HCC.

\section{Levels of coenzyme Q10, antioxidant capacity, and inflammation after supplementation}

The levels of coenzyme Q10, vitamin E, oxidative stress, and antioxidant enzymes after intervention are shown in Table 2 and Fig. 2. The plasma coenzyme Q10 concentration was significantly increased after the coenzyme Q10 supplementation $(P<0.01)$. Subjects in the Q10-300 group had significantly higher levels of coenzyme Q10 than those in the placebo group at weeks 4,8 , and $12(P<0.01)$. The plasma MDA level was significantly decreased after the coenzyme Q10 supplementation $(1.48 \pm 0.58 \mu \mathrm{M}$ decreased to $1.22 \pm 0.31 \mu \mathrm{M}, P=0.04)$ and in the patients in the

Table 2 Changes in the levels of coenzyme Q10, vitamin E, oxidative stress, antioxidant enzymes activities, and inflammation in HCC patients after supplementation

\begin{tabular}{|c|c|c|c|c|c|c|}
\hline & \multicolumn{3}{|l|}{ Placebo $(n=19)$} & \multicolumn{3}{|l|}{ Q10-300 $(n=20)$} \\
\hline & Week 0 & Week 12 & Week $12-0^{b}$ & Week 0 & Week 12 & Week 12-0 \\
\hline coenzyme Q10 ( $\mu \mathrm{M})$ & $0.32 \pm 0.14(0.33)^{a}$ & $0.38 \pm 0.09(0.37)$ & $0.11 \pm 0.15(0.09)$ & $0.27 \pm 0.08(0.27)$ & $1.47 \pm 0.82(1.58)^{e, d}$ & $1.19 \pm 0.84(1.35)^{d}$ \\
\hline vitamin $\mathrm{E}(\mu \mathrm{M})$ & $11.84 \pm 2.98(11.66)$ & $12.04 \pm 4.13(11.47)$ & $0.20 \pm 2.79(0.51)$ & $11.16 \pm 2.20(12.10)$ & $11.52 \pm 3.28(10.54)$ & $0.43 \pm 2.29(0.54)$ \\
\hline \multicolumn{7}{|l|}{ Oxidative stress } \\
\hline $\mathrm{MDA}(\mu \mathrm{M})$ & $1.37 \pm 0.34(1.42)$ & $1.41 \pm 0.30(1.37)$ & $0.08 \pm 0.35(0.11)$ & $1.48 \pm 0.58(1.33)$ & $1.22 \pm 0.31(1.10)^{e, d}$ & $-0.27 \pm 0.68(-0.33)^{d}$ \\
\hline \multicolumn{7}{|c|}{ Antioxidant enzymes activity } \\
\hline SOD (U/mg protein) & $16.94 \pm 8.78(13.10)$ & $17.12 \pm 5.79(16.90)$ & $0.73 \pm 11.37(3.00)$ & $13.93 \pm 4.35(13.16)$ & $21.26 \pm 7.86(22.02)^{e, d}$ & $7.96 \pm 7.15(7.50)^{d}$ \\
\hline CAT (U/mg protein) & $16.36 \pm 7.97(12.00)$ & $15.24 \pm 6.62(12.80)$ & $-1.71 \pm 6.25(0.29)$ & $14.74 \pm 7.63(11.75)$ & $18.00 \pm 6.82(16.76)^{\mathrm{e}, \mathrm{d}}$ & $3.28 \pm 7.76(4.80)^{d}$ \\
\hline GPx (U/mg protein) & $15.22 \pm 5.76(14.66)$ & $12.73 \pm 5.46(12.20)$ & $-1.10 \pm 7.26(-0.60)$ & $15.94 \pm 4.77(17.46)$ & $19.40 \pm 8.34(22.08)^{e, d}$ & $4.08 \pm 7.67(4.70)^{d}$ \\
\hline \multicolumn{7}{|l|}{ Inflammatory markers } \\
\hline hs-CRP (mg/L) & $3.5 \pm 3.6(1.6)$ & $2.1 \pm 3.9(0.8)$ & $-1.5 \pm 4.2(-0.8)$ & $4.2 \pm 4.2(2.8)$ & $2.6 \pm 4.2(1.0)^{\mathrm{e}}$ & $-1.7 \pm 4.8(-1.3)$ \\
\hline TNF-a (pg/mL) & $0.89 \pm 0.71(1.16)$ & $1.77 \pm 4.38(0.73)$ & $0.88 \pm 4.59(-0.10)$ & $0.84 \pm 0.78(0.73)$ & $1.06 \pm 1.02(0.86)$ & $0.30 \pm 1.19(0.02)$ \\
\hline IL-6 (pg/mL) & $3.76 \pm 2.30(3.44)$ & $2.83 \pm 3.64(1.34)$ & $-1.05 \pm 3.72(-1.65)$ & $3.07 \pm 2.03(1.98)$ & $1.62 \pm 0.75(1.70)^{\mathrm{e}}$ & $-0.49 \pm 2.96(-0.70)$ \\
\hline
\end{tabular}

CAT Catalase activity, GPx glutathione peroxidase, HCC hepatocellular carcinoma, $h s-C R P$ high sensitivity C-reactive protein, IL-6 interleukin-6, MDA Malondialdehyde, $S O D$ superoxide dismutase, TNF-a tumor necrosis factor-a

ameans \pm SD (medians)

${ }^{b}$ Difference between week 12 and week 0

${ }^{d}$ values are significantly different between the two groups

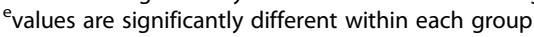



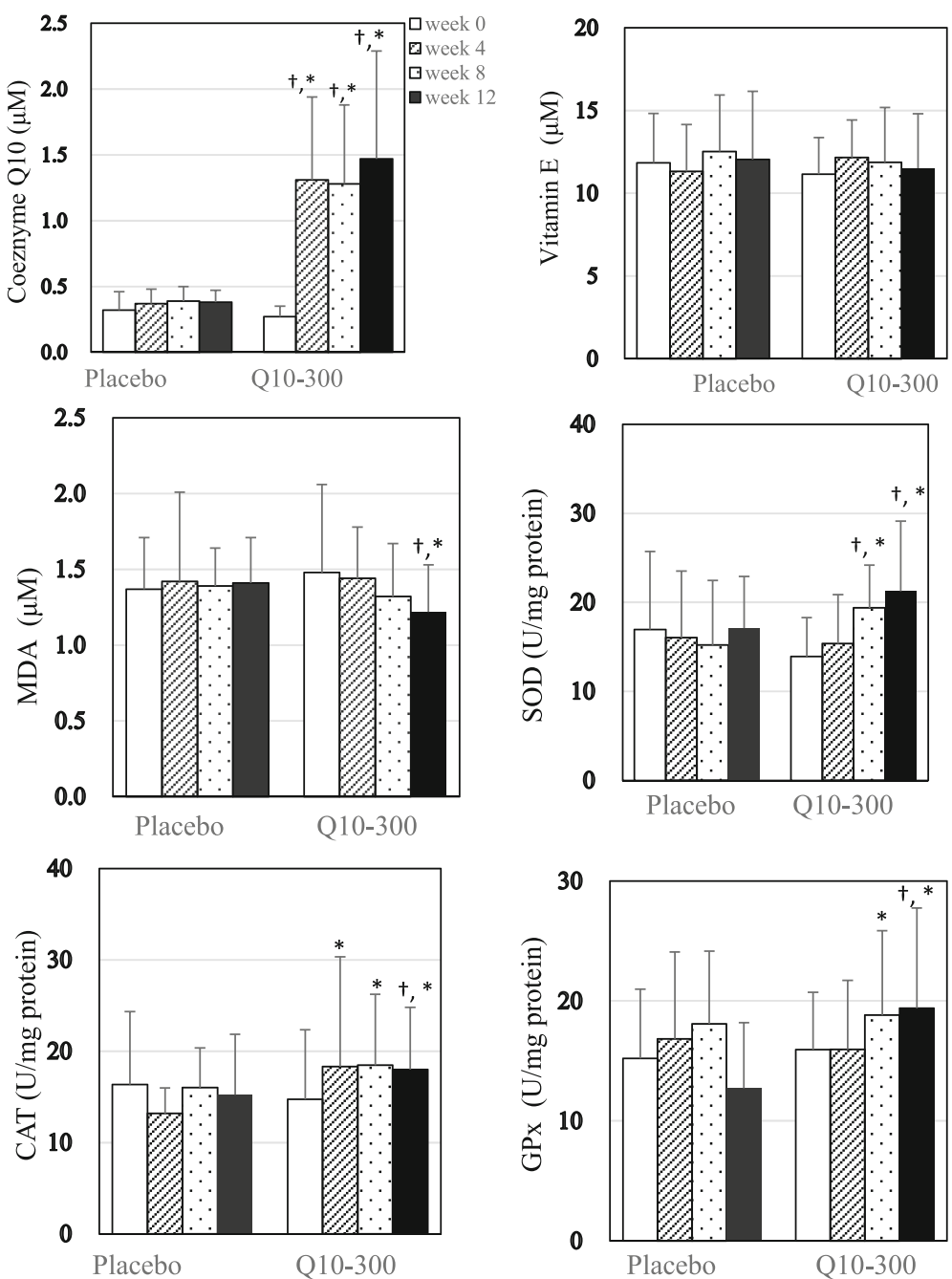

Fig. 2 Levels of coenzyme Q10, vitamin E, oxidative stress, and antioxidant enzymes after supplementation. ${ }^{\dagger}$ values are significantly different between the two groups. "values are significantly different within each group. CAT, catalase activity; GPx, glutathione peroxidase; HCC, hepatocellular carcinoma; MDA, malondialdehyde; SOD, superoxide dismutase

placebo group at week $12(1.22 \pm 0.31 \mu \mathrm{M}$ vs. $1.41 \pm$ $0.30 \mu \mathrm{M}, P=0.04)$. In terms of the antioxidant enzymes activity, the SOD $(13.93 \pm 4.35 \mathrm{U} / \mathrm{mg}$ protein increased to $19.39 \pm 4.79 \mathrm{U} / \mathrm{mg}$ protein, $P<0.01)$ and GPx activities $(15.94 \pm 4.77 \mathrm{U} / \mathrm{mg}$ protein increased to $18.82 \pm 7.03 \mathrm{U} /$ mg protein, $P<0.01)$ were significantly increased after 8 weeks of coenzyme Q10 supplementation and the CAT activity was significantly increased after 4 weeks of coenzyme Q10 supplementation $(14.74 \pm 7.63 \mathrm{U} / \mathrm{mg}$ protein increased to $18.32 \pm 12.03 \mathrm{U} / \mathrm{mg}$ protein, $P$ $<0.01)$.

The post-supplementation changes in the levels of coenzyme Q10, vitamin $\mathrm{E}$, oxidative stress, antioxidant enzymes activities, and inflammation in HCC patients are also shown in Table 2. After 12 weeks of supplementation, subjects in the Q10-300 supplementation showed an increased level of changes in their level of coenzyme Q10 $(P<0.01)$ and antioxidant enzymes activity (SOD, $P=0.03$; CAT, $P=0.01$; GPx, $P<0.01$ ) than those in the placebo group. The levels of MDA $(P<0.01)$ and inflammatory markers were significantly lower in the Q10-300 group compared to the placebo group after 12 weeks of supplementation (hs-CRP, $4.2 \pm 4.2 \mathrm{mg} / \mathrm{L}$ decreased to $2.6 \pm 4.2 \mathrm{mg} / \mathrm{L}, P<0.01$; IL-6, $3.07 \pm 2.03 \mathrm{pg} /$ $\mathrm{mL}$ decreased to $1.62 \pm 0.75 \mathrm{pg} / \mathrm{mL}, P<0.01)$. However, the levels of vitamin $\mathrm{E}(P=0.81)$ and inflammatory markers (hs-CRP, $P=0.64$, TNF- $\alpha, P=0.68$, IL-6, $P=$ 0.80 ), as well as the difference between week 12 and week 0 of inflammatory markers (hs-CRP, $P=0.58$, TNF- $\alpha, P=0.29$, IL-6, $P=0.13$ ) were not significantly different between the placebo and Q10-300 groups after supplementation. 


\section{Correlations between coenzyme Q10, antioxidant capacity, and inflammation after supplementation} The correlations between coenzyme Q10, vitamin E, oxidative stress, antioxidant enzymes activities, and inflammation after supplementation are shown in Table 3. The plasma coenzyme Q10 concentration was significantly correlated with the levels of vitamin E $(r=0.30$, $P=0.01)$, MDA $(r=-0.43, P=0.01)$, and antioxidant enzymes activities (SOD, $r=0.31, P=0.01$; CAT, $r=0.24$, $P<0.05$; GPx, $r=0.25, P=0.04$ ) after 12 weeks of supplementation. However, there was no significant association between the levels of coenzyme Q10 and inflammatory markers after supplementation.

Furthermore, we examined the correlations between the changes in the levels of coenzyme Q10 and oxidative stress, antioxidant enzymes, and vitamin E (Fig. 3). Changes in the levels of coenzyme Q10 was significantly correlated with changes in the levels of MDA $(\beta=-0.29, \quad p=0.02)$, antioxidant enzymes activity (SOD, $\beta=2.57, p=0.02$; CAT, $\beta=4.47, p<0.01 ; \beta=$ 3.53, $p=0.03)$, and vitamin $\mathrm{E}(\beta=1.44, p=0.01)$.

\section{Discussion}

This is the first clinical study to demonstrate that coenzyme Q10 (at a dose of $300 \mathrm{mg} / \mathrm{d}$ for 12 weeks) increases antioxidant enzymes activities (SOD, CAT, and GPx) and decreases oxidative stress and inflammation in patients with HCC. Coenzyme Q10 shows a significant anticarcinogenic activity in various cancer models via its antioxidant and anti-inflammatory properties $[10,11,18,19]$. Coenzyme Q10 is a highly safe and well tolerable supplement. Because coenzyme Q10 is a well

Table 3 Correlations between coenzyme Q10 level and vitamin E, oxidative stress, antioxidant enzymes activity and inflammation in HCC patients after supplementation

\begin{tabular}{ll}
\hline & $\begin{array}{l}\text { Coenzyme Q10 level }(\mu \mathrm{M}) \\
r^{2}(p \text { values })\end{array}$ \\
\hline vitamin $\mathrm{E}(\mu \mathrm{M})$ & $0.30(0.01)$ \\
Oxidative stress & $-0.43(0.01)$ \\
MDA $(\mu \mathrm{M})$ & \\
Antioxidant enzymes activity & $0.31(0.01)$ \\
SOD $(\mathrm{U} / \mathrm{mg}$ protein) & $0.24(<0.05)$ \\
CAT $(\mathrm{U} / \mathrm{mg}$ protein) & $0.25(0.04)$ \\
GPx $(\mathrm{U} / \mathrm{mg}$ protein) & \\
Inflammatory markers & $0.12(0.49)$ \\
hs-CRP $(\mathrm{mg} / \mathrm{L})$ & $0.01(0.96)$ \\
TNF-a $(\mathrm{pg} / \mathrm{mL})$ & $0.00(0.99)$ \\
IL-6 $(\mathrm{pg} / \mathrm{mL})$ &
\end{tabular}

CAT Catalase activity, MDA Malondialdehyde, GPx glutathione peroxidase, HCC hepatocellular carcinoma, $h s-C R P$ high sensitivity C-reactive protein, IL-6 interleukin-6, SOD superoxide dismutase, TNF-a tumor necrosis factor-a ${ }^{a}$ correlation coefficient antioxidant and anti-inflammation agent, it may represent a potential complementary therapeutic option in chronic disease patients who suffer from a higher status of oxidative stress or inflammation [20-22].

In this study, subjects in both groups had a low level of coenzyme Q10 (normal ranges: 0.5-1.7 $\mu \mathrm{M}$ ) [23] at baseline $(0.30 \pm 0.11 \mu \mathrm{M})$. After a 4-week supplementation of coenzyme Q10 supplementation at a dose of $300 \mathrm{mg} / \mathrm{d}$, the low level of coenzyme Q10 rapidly and significantly increased in patients with HCC $(0.27 \pm$ $0.08 \mu \mathrm{M}$ to $1.31 \pm 0.63 \mu \mathrm{M}, p<0.01)$, and this level was sustained for 12 weeks. Additionally, antioxidant enzyme activity significantly increased after the coenzyme Q10 supplementation, the CAT activity was significantly increased by $25.5 \%$ after 4 weeks of supplementation, and SOD, CAT, and GPx were significantly increased by $43.2 \%, 40.4 \%$, and $13.4 \%$ after 8 weeks of supplementation. After 12 weeks of coenzyme Q10 supplementation, SOD, CAT, and GPx were significantly increased by $67.3 \%, 42.6 \%$, and $26.5 \%$ and oxidative stress (MDA) was significantly decreased by $17.3 \%$. Although there was no significant difference in the level of vitamin $\mathrm{E}$ after 12 weeks of supplementation, there was a significantly positive correlation between the levels of coenzyme Q10 and vitamin E after supplementation. Because patients with HCC suffer from a higher level of oxidative stress and inflammation [5], and a deficiency in coenzyme Q10 was found in HCC patients, we suggest that the administration of coenzyme Q10 supplements in patients with HCC during the surgery may be beneficial. Administered coenzyme Q10 at dose of $300 \mathrm{mg} / \mathrm{d}$ to HCC patients can significantly increase their antioxidant capacity, protect vitamin E against superoxide-driven oxidation and regenerate/maintain the level of vitamin $\mathrm{E}$ during antioxidation processes [24, 25].

Patients infected with hepatitis $\mathrm{B}$ and hepatitis $\mathrm{C}$ viruses account for $65-80 \%$ of HCC worldwide [26]. In the present study, 66.7 and $28.2 \%$ of the patients with HCC had a history of infection with hepatitis B and hepatitis $\mathrm{C}$ viruses, respectively. These chronic infections cause liver inflammation, and may lead to liver fibrosis/ cirrhosis or HCC [27]. In this study, a total of $69.2 \%$ of the subjects $(N=27$, placebo, $n=11$; Q10-300, $n=16)$ had chronic inflammation (hs-CRP $\geq 1.0 \mathrm{mg} / \mathrm{L}$ ) and $38.5 \%$ of the subjects $(N=15$, placebo, $n=5$; Q10-300, $n=10$ ) had a higher level of inflammation (hs-CRP $\geq$ $3.0 \mathrm{mg} / \mathrm{L}$ ) after surgery. After 12 weeks of coenzyme Q10 supplementation, the levels of hs-CRP and IL-6 were significantly decreased (Table 2), although the levels of inflammatory markers were not significantly lower compared to the placebo group and no significant association between the level of coenzyme Q10 and inflammatory markers after intervention (Table 3). We failure to detect significant correlations between the 
A

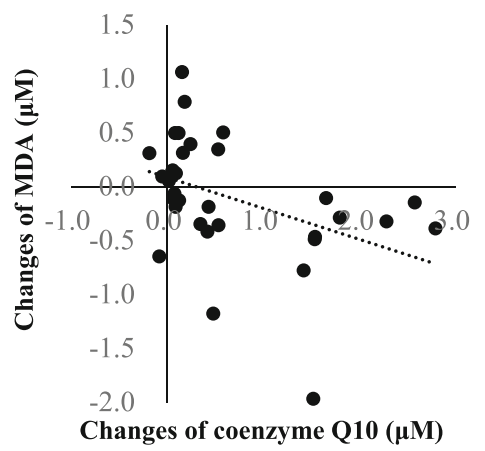

C

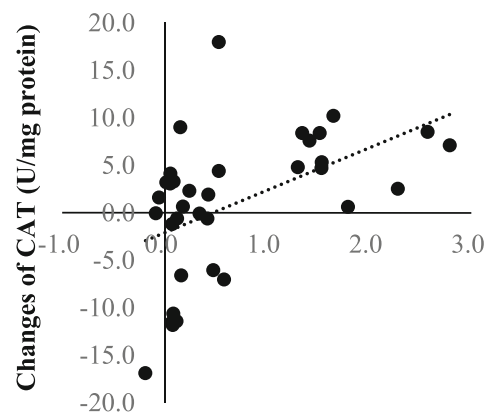

B

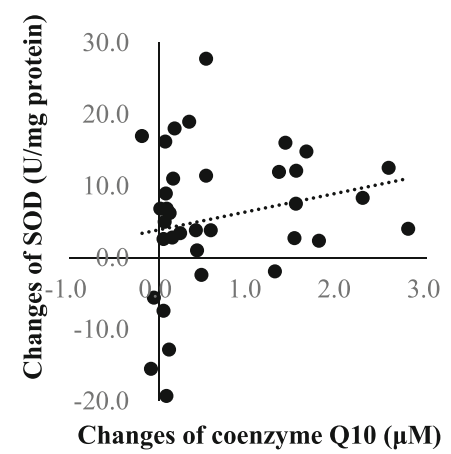

D

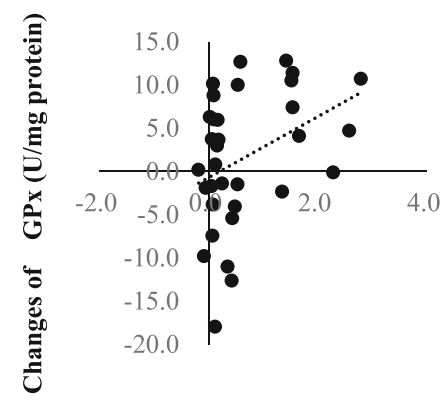

Changes of coenzyme Q10 $(\mu \mathrm{M})$

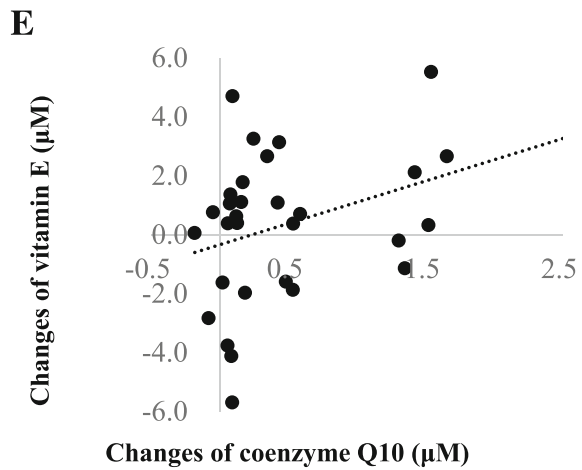

Fig. 3 Correlations between changes in the levels of coenzyme Q10 and changes in the levels of oxidative stress, antioxidant enzymes activity, and vitamin E. a Correlation between the changes in the levels of coenzyme Q10 and MDA $(\beta=-0.29, p=0.02)$; $\mathbf{b}$ Correlation between the changes in the levels of coenzyme Q10 and SOD $(\beta=2.57, p=0.02)$; c Correlation between the changes in the levels of coenzyme Q10 and CAT $(\beta=4.47, p<0.01)$; $\mathbf{d}$ Correlation between the changes in the levels of coenzyme Q10 and GPx $(\beta=3.53, p=0.03)$; e Correlation between the changes in the levels of coenzyme Q10 and vitamin $\mathrm{E}(\beta=1.44, p=0.01)$. CAT, catalase activity; GPT, glutamic oxaloacetic transaminase; GPX, glutathione peroxidase; HCC, hepatocellular carcinoma; MDA, malondialdehyde; SOD, superoxide dismutase

levels of coenzyme Q10 and inflammatory markers maybe due to higher variations of inflammation status in patients with HCC after surgery. Surgical resection is the most efficient treatment of patients with HCC. However, surgery may elicits the activation of the systemic inflammatory, endocrine/metabolic, and immunological systems, which is referred to as the surgical stress response [28]. However, Coenzyme Q10, as an antioxidant, could inhibit the inflammatory cascade of NF- $k B$-activation by the ROS [29-31]. Therefore, it may be useful to decrease inflammation in patients with HCC after surgery through coenzyme Q10 to increase the antioxidant capacity, particularly those with a higher level of inflammation.

Regarding the safety of coenzyme Q10 supplementation in the present study, there were no clinically significant changes in the subjects' vital signs, serum chemical values, or hematological values, and there were no serious adverse events, no complaints of myalgia or muscle weakness, and no withdrawals due to adverse events. 
Note that the level of GPT was significantly decreased after the coenzyme Q10 supplementation (data not shown, $55.5 \pm 39.8 \mathrm{IU} / \mathrm{L}$ decreased to $49.1 \pm 46.2 \mathrm{IU} / \mathrm{L}, p$ $=0.02$ ) and that changes in the levels of coenzyme Q10 were significantly correlated with changes in the levels of GPT (data not shown, $\beta=-10.09, p=0.03$ ). In addition to GPT, the level of high density lipoproteincholesterol (HDL-C) was significantly increased after the coenzyme Q10 supplementation (data not shown, $1.3 \pm$ $0.3 \mathrm{mmol} / \mathrm{L}$ increased to $1.5 \pm 0.4 \mathrm{mmol} / \mathrm{L}, p<0.01)$. Thus, at a dose of $300 \mathrm{mg} / \mathrm{d}$ coenzyme Q10 is safe for patients with $\mathrm{HCC}$, and it protects against cardiotoxicity or liver toxicity during cancer treatment [32]. Our study has two limitations should be mentioned. First, the number of participants was small, although we did recruit more subjects than expected. Second, this study was designed for 12 weeks intervention only. Larger and longer intervention studies are needed to confirm the beneficial effect of coenzyme Q10 supplementation in patients with HCC after surgery.

\section{Conclusion}

In conclusion, this clinical study is the first to demonstrate that a dose of $300 \mathrm{mg} / \mathrm{d}$ of coenzyme Q10 supplementation significantly increased antioxidant capacity and reduced the levels of inflammatory markers (hs-CRP and IL-6) in patients with HCC after surgery. We suggest that coenzyme Q10 supplementation could be considered as a complementary treatment strategy for patients with HCC after surgery, particularly those under higher levels of oxidative stress and inflammation.

\section{Acknowledgments \\ We would like to express our sincere appreciation to the subjects for their participation and to Dr. Hsia, who kindly provided the coenzyme Q10 supplements for this trial. We thank the nurses at Taichung Veterans General Hospital and the technical advisor at the Taipei Institute of Pathology for providing expert assistance in blood sample collection and data analysis.}

\section{Funding}

This study was supported by a grant from the Taichung Veterans General Hospital (TCVGH-1034601A and TCVGH-1044601A), Taiwan.

\section{Availability of data and materials}

This study acts as a pilot study for a larger study that will be performed. Data sets will be made available once the results of the larger study is published. However those who are interesting to get the data can contact PTL: apt810@csmu.edu.tw for further information.

\section{Authors' contributions}

HTL, YCH, and SBJ performed the study and the data analyses. YTH helped to perform out the study and sample analyses. PTL conceived of the study, participated in its design, and coordinated the study. HTL and PTL drafted the manuscript. All authors read and approved the final manuscript.

\section{Competing interests}

The authors declare that they have no competing interests.

\section{Consent for publication}

Not applicable.

\section{Ethics approval and consent to participate}

This study was approved by the Institutional Review Board of Taichung Veterans General Hospital, Taiwan and each subject gave written informed consent before participating. All protocols and procedures were performed according to the Declaration of Helsinki. This study was registered under NCT01964001 at Clinicaltrials.gov.

\section{Author details}

${ }^{1}$ Graduate Program in Nutrition, Chung Shan Medical University, Taichung 40201, Taiwan. ${ }^{2}$ Division of General Surgery, Department of Surgery, Taichung Veterans General Hospital, Taichung 40705, Taiwan. ${ }^{3}$ Department of Nutrition, Chung Shan Medical University, Taichung 40201, Taiwan.

${ }^{4}$ Department of Nutrition, Chung Shan Medical University Hospital, Taichung 40201, Taiwan.

Received: 15 August 2016 Accepted: 3 October 2016

Published online: 06 October 2016

\section{References}

1. Stewart BW, Wild CP. World Cancer Report 2014. Geneva: IARC Nonserial Publication; 2014.

2. El-Serag HB. Hepatocellular carcinoma. N Engl J Med. 2011;365:1118-27.

3. Akinobu T, Kazuhide Y. Control of oxidative stress in hepatocellular carcinoma: Helpful or harmful? World J Hepatol. 2015;7:968-79.

4. Muriel P. Role of free radicals in liver diseases. Hepatol Int. 2009;3:526-36.

5. Yahya RS, Ghanem OH, Foyouh AA, Atwa M, Enany SA. Role of interleukin-8 and oxidative stress in patients with hepatocellular carcinoma. Clin Lab. 2013:59:969-76.

6. Ernster L, Dallner G. Biochemical, physiological and medical aspects of ubiquinone function. Biochim Giophys Acta. 1995;1271:195-204.

7. Bhagavan HN, Chopra RK. Coenzyme Q10: absorption, tissue uptake, metabolism and pharmacokinetics. Free Radic Res. 2006;40:445-53.

8. Alleva R, Tomasetti M, Battino M, Curatola G, Littarru GP, Folkers K. The roles of coenzyme Q10 and vitamin E on the peroxidation of human low density lipoprotein subfractions. Proc Natl Acad Sci U S A. 1995;26:9388-91.

9. $\quad$ Singh U, Devaraj S, Jialal I. Coenzyme Q10 supplementation and heart failure. Nutr Rev. 2007;1:286-93.

10. Fouad AA, Al-Mulhim AS, Jresat I. Therapeutic effect of coenzyme Q10 against experimentally-induced hepatocellular carcinoma in rats. Environ Toxicol Pharmacol. 2013;35:100-8.

11. Kim JM, Park E. Coenzyme Q10 attenuated DMH-induced precancerous lesions in SD rats. J Nutr Sci Vitaminol. 2010;56:139-44.

12. Karpińska J, Mikołuć B, Motkowski R, Piotrowska-Jastrzebska J. HPLC method for simultaneous determination of retinol, alpha-tocopherol and coenzyme Q10 in human plasma. J Pharm Biomed Anal. 2006:42:232-6.

13. Littarru GP, Mosca F, Fattorini D, Bompadre S. Method to assay coenzyme Q10 in blood plasma or blood serum. United State Patent. 2007; 7303921. https://patentimages.storage.googleapis.com/pdfs/6aadab0bc819eac3443b/ US7303921.pdf.

14. Botsoglou NA. Rapid, sensitive, and specific thiobarbituric acid method for measuring lipid peroxidation in animal tissue, food and feedstuff samples. J Agric Food Chem. 1994;42:1931-7.

15. Paglia D, Valentine W. Studies on the qualitative characterization of erythrocyte glutathione peroxidase. J Lab Clin Med. 1967;70:159-69.

16. Marklund S, Marklund G. Involvement of superoxide anion radical in autoxidation of pyrogallol and a convenient assay for superoxide dismutase. Eur J Biochem. 1974;47:469-74.

17. Aebi H. Catalase in vitro. Methods Enzymol. 1984;105:121-6.

18. Sakano K, Takahashi M, Kitano M, Sugimura T, Wakabayashi K. Suppression of azoxymethane-induced colonic premalignant lesion formation by coenzyme Q10 in rats. Asian Pac J Cancer Prev. 2006;7:599-603.

19. Bahar M, Khaghani S, Pasalar P, Paknejad M, Khorramizadeh MR, Khorramizadeh MR, Mirmiranpour H, Nejad SG. Exogenous coenzyme Q10 modulates MMP-2 activity in MCF-7 cell line as a breast cancer cellular model. Nutr J. 2010;9:62

20 Lee BJ, Huang YC, Chen SJ, Lin PT. Coenzyme Q10 supplements reduce oxidative stress and increase activities of antioxidant enzymes in patients with coronary artery disease. Nutrition. 2012;28:250-5.

21 Lee BJ, Huang YC, Chen SJ, Lin PT. Effects of coenzyme Q10 supplementation on inflammatory markers (high sensitivity C-reactive 
protein, interleukin- 6 and homocysteine) in patients with coronary artery. Nutrition. 2012;28:767-72.

22 Lee BJ, Tseng YF, Yen CH, Lin PT. Effects of coenzyme Q10 supplementation (300 mg/day) on antioxidation and anti-inflammation in coronary artery disease patients during statins therapy: a randomized, placebo-controlled trial. Nutr J. 2013;12:142.

23 Molyneux SL, Young JM, Florkowski CM, Lever M, Georgr PM. Coenzyme Q10: Is there a clinical role and a case for measurement? Clin Biochem Rev. 2008:29:71-82

24 Stoyanovsky DA, Osipov AN, Quinn PJ, Kagan VE. Ubiquinone-dependent recycling of vitamin E radicals by superoxide. Arch Biochem Biophys. 1995: 323:343-51.

25 Lass A, Sohal RS. Electron transport-linked ubiquinone-dependent recycling of a-tocopherol inhibits autooxidation of mitochondrial membranes. Arch Biochem Biophys. 1998:352:229-36.

26 Bosch FX, Ribes J, Borràs J. Epidemiology of primary liver cancer. Semin Liver Dis. 1999;19:271-85.

27 Boltjes A, Movita D, Boonstra A, Woltman AM. The role of Kupffer cells in hepatitis B and hepatitis C virus infections. J Hepatol. 2014;61:660-71.

28 Kücükakin B, Gögenur I, Reiter RJ, Rosenberg J. Oxidative stress in relation to surgery: is there a role for the antioxidant melatonin? J Surg Res. 2009;152: 338-47.

29 Schmelzer C, Lindner I, Rimbach G, Niklowitz P, Menke T, Döring F. Functions of coenzyme Q10 in inflammation and gene expression. Biofactors. 2008;32:179-83.

30 Schmelzer C, Lorenz G, Rinbach G, Doring F. In vitro effects of the reduced form of coenzyme Q(10) on secretion levels of TNF-alpha and chemokines in response to LPS in the human monocytic cell line THP-1. J Clin Biochem Nutr. 2009;44:62-6.

31 Zhang YP, Eber A, Yuan Y, Yang Z, Rodriguez Y, Levitt RC, Takacs P, Candiotti KA. Prophylactic and antinociceptive effects of coenzyme Q10 on diabetic neuropathic pain in a mouse model of type 1 diabetes. Anesthesiology. 2013;118:945-54.

32 Roffe L, Schmidt K, Ernst E. Efficacy of coenzyme Q10 for improved tolerability of cancer treatments: a systematic review. J Clin Oncol. 2004;22: 4418-24.

\section{Submit your next manuscript to BioMed Central and we will help you at every step:}

- We accept pre-submission inquiries

- Our selector tool helps you to find the most relevant journal

- We provide round the clock customer support

- Convenient online submission

- Thorough peer review

- Inclusion in PubMed and all major indexing services

- Maximum visibility for your research

Submit your manuscript at www.biomedcentral.com/submit 